\section{Psychological Medicine}

cambridge.org/psm

\section{Review Article}

Cite this article: Hedman-Lagerlöf $M$, Hedman-Lagerlöf E, Öst L-G (2018). The empirical support for mindfulness-based interventions for common psychiatric disorders: a systematic review and meta-analysis. Psychological Medicine 48, 2116-2129. https://doi.org/10.1017/ S0033291718000259

Received: 7 May 2017

Revised: 17 January 2018

Accepted: 17 January 2018

First published online: 19 February 2018

\section{Key words:}

Clinical efficacy; common psychiatric disorders; evidence status; meta-analysis; mindfulness-based interventions

Author for correspondence:

Maria Hedman-Lagerlöf,

E-mail: maria.hedman-lagerlof@ki.se

\title{
The empirical support for mindfulness-based interventions for common psychiatric disorders: a systematic review and meta-analysis
}

Maria Hedman-Lagerlöf ${ }^{1}$, Erik Hedman-Lagerlöf ${ }^{1}$ and Lars-Göran Öst ${ }^{2}$

${ }^{1}$ Department of Clinical Neuroscience, Division of Psychology, Karolinska Institutet, Stockholm, Sweden and

${ }^{2}$ Department of Psychology, Stockholm University, Stockholm, Sweden

\section{Abstract}

Mindfulness-based interventions (MBIs) have become widely used for common mental disorders (CMDs) but the state of the evidence has not been sufficiently investigated. The aims for this study were: (1) to quantify the effect size of MBIs for CMDs in the acute phase; (2) to explore moderator variables; and (3) to evaluate the evidence status of MBIs for the CMDs it has been tried for. A comprehensive systematic review and meta-analysis of randomized controlled trials (RCTs) were conducted. RCTs that evaluated MBI and included patients with a primary manifest CMD was included. Methodological quality, the risk of bias, publication bias and evidence status were assessed. Literature searches gave 2448 hits and 19 studies were included. MBIs were more effective than no treatment $(g=1.07)$ and treatment-as-usual $(g=0.40)$ but not in comparison to placebo $(g=0.17)$ or other active treatments $(g=-0.01)$. Methodological quality was negatively correlated with outcome. For all psychiatric disorders it has been tested, MBIs were judged to have weak or no empirical support. The conclusion of the study is that the evidence-base for MBIs for CMDs in the acute phase is weak.

\section{Introduction}

During the last 20 years, research on mindfulness-based interventions (MBIs) as a potential treatment for several common mental health problems has increased substantially (Baer, 2003; Brown et al. 2007; Ludwig \& Kabat-Zinn, 2008). Originally developed for coping with the stress of chronic illness (Kabat-Zinn, 1996), MBIs have also shown to be an effective relapse treatment for depression (Teasdale et al. 2000; Segal et al. 2010; Williams et al. 2014), a common mental disorder (CMD). MBIs could potentially be a promising treatment for other CMDs also in the acute phase, which has been suggested, e.g. by Kearney et al. (2013), Koszycki et al. (2007) and McManus et al. (2012). In line with this, there now seems to be a growing trend within regular healthcare to implement and offer MBIs as a treatment to patients with common mental disorders (CMDs) such as depression and anxiety disorders (e.g., Plank, 2010; Demarzo et al. 2015a; Dimidjian \& Segal, 2015).

Given that MBIs have gained popularity and considering the large burden of disease caused by psychiatric disorders (Degenhardt et al. 2013), it is important to establish the empirical support for MBIs in the treatment of CMDs in the acute phase. Several meta-analyses and literature reviews have been published in the last decade evaluating the effect of MBIs on psychiatric and somatic ill-health (e.g., Hofmann, Sawyer, Witt, \& Oh, 2010; Chiesa \& Serretti, 2011; Piet \& Hougaard, 2011; Vollestad, Nielsen, \& Nielsen, 2012; Khoury et al. 2013; Goyal et al. 2014; Strauss, Cavanagh, Oliver, \& Pettman, 2014; Demarzo et al. 2015b). Although results have been somewhat mixed, several meta-analyses have concluded that MBIs are a promising treatment for reducing anxiety, stress and preventing depressive relapse (e.g., Hofmann et al. 2010; Piet \& Hougaard, 2011; Vollestad et al. 2012; Khoury et al. 2013; Goyal et al. 2014). However, to our knowledge, no study has previously been published that investigated the aggregated effect of MBIs as a stand-alone treatment of CMDs in the acute phase (i.e., not as a relapse prevention). Also, the methodology used in previous meta-analyses has not yet permitted this question to be adequately addressed. Firstly, a number of them have included studies in which the samples have not been patients with a psychiatric disorder as the principal problem. More specifically, a majority of randomized controlled trials (RCTs) in this field have studied the effect of MBIs on symptoms of stress, sleep problems, anxiety or depression in patients with somatic disorders (e.g., Lengacher et al. 2009; Gross et al. 2010; Bränström, Kvillemo, \& Moskowitz, 2012; Bower et al. 2015) or have included patients based on self-reported symptoms without using reliable instruments to assess the target disorder in the recruitment phase (e.g., Biegel, Brown, Shapiro, \& Schubert, 2009; Delgado 
et al. 2010; Black et al. 2015). Secondly, no previous meta-analysis, except one by Strauss et al. (2014), has investigated the effect of MBIs as a stand-alone treatment for psychiatric disorders where all forms of MBIs have been included. The previous meta-analysis by Strauss et al. (2014) however only included studies evaluating MBIs for depressive- and anxiety disorders, thus excluding studies evaluating the effect on for example insomnia and adjustment disorder. Some meta-analyses have focused solely on mindfulness-based stress reduction (MBSR; e.g. Grossman, Niemann, Schmidt, \& Walach, 2004) or mindfulness-based cognitive therapy (MBCT; e.g., Chiesa \& Serretti, 2011; Galante, Iribarren, \& Pearce, 2013; Lenz et al. 2016), excluding studies evaluating other MBIs. Other meta-analyses have had the opposite limitation, i.e. the isolated effect of MBIs could not be determined due to the inclusion of other meditation interventions in addition to mindfulness (e.g., Vollestad et al. 2012; Goyal et al. 2014). Thirdly, most previous meta-analyses specifically evaluating the effect of MBCT (Chiesa \& Serretti, 2011; Piet \& Hougaard, 2011; Galante et al. 2013) have investigated prevention of relapse in depression with the notable exception of Lenz et al. (2016); who concluded that MBCT was effective in reducing symptoms of acute depression. However, it remains unclear if other MBIs are effective in reducing depressive symptoms in a clinically manifest disorder in the acute phase. Lastly, to the best of our knowledge, there is only one previous meta-analysis (Goyal et al. 2014) that has evaluated MBIs against stringent and recognized criteria for evidence-based treatments.

To address the limitations in the previous literature, we conducted a comprehensive systematic review and meta-analysis of MBIs for manifest common psychiatric disorders in the acute phase (i.e., prevention studies and studies evaluating relapse prevention were not included), with the following objectives: (1) to quantify the size of the treatment effect; (2) to explore moderator variables contributing to the treatment effect; and (3) to evaluate the evidence status of MBIs for the different psychiatric disorders it has been tried for. Outcome measures were symptoms of the respective principal disorder. We compared MBIs with waitlist control, no-treatment control, placebo and active psychological and pharmacological treatments. Only RCTs were included. The study is reported following the Preferred Reporting Items for Systematic Reviews and Meta-analyses (PRISMA) guidelines (Moher et al. 2009).

\section{Method}

\section{Literature search}

Studies were identified using PubMed, Cinahl, Scopus, Psychinfo, and Cochrane databases from when first available until February 1,2016 . We used the search terms mindful ${ }^{*}$, MBSR, MBCT, either combined with the terms random* or restricted to clinical trials and RCTs, and restricted to the English language where applicable. We did not include search terms pertaining to the targeted disorders (e.g., anxiety and depression ${ }^{\star}$ ), thus accepting a high number of articles to read to minimize the risk of missing relevant studies. Search strings were formulated separately for each database in free text. Subject headings or MeSH-terms were used where applicable. Reference lists of published meta-analyses and reviews were also screened to identify potential studies of relevance. See online Other Supplementary Material (OSM), Figure OSM1 for an example of a full search strategy.

\section{Study selection}

Table 1 displays the criteria that studies had to meet to be included and Figure 1 displays the flowchart of the selection process. After removal of duplicates, the first author screened all titles and abstracts. Studies that were clearly irrelevant were excluded and those who were potentially relevant were retrieved in full text and assessed for eligibility. In ambiguous cases, attempts were made to contact the authors of the study in question. Cases, where it was uncertain if inclusion criteria were met, were resolved by discussion with the co-authors.

\section{Data extraction process}

Data regarding study background variables were extracted regarding research design (e.g., control condition and length of follow-up), sample (e.g., mean age, $\%$ attrition, $\%$ decliners and $\%$ comorbidity) and intervention (e.g., length of intervention, therapist quality and therapy format). Variables were extracted as continuous (e.g., length of follow-up, per cent women and mean age) or categorical (e.g., control condition, disorder and country) as applicable. Means and standard deviations (s.D.) of outcome measures at pre-, post and follow-up (when available) were extracted for meta-analytical calculations. We also coded several variables of the study as potential moderator variables, based on the literature in the field. A full list of the variables extracted is presented in the OSM (online Table OSM1). The data were extracted from each study independently by the first author. Any uncertainties were discussed with the last author and contact attempts were made to obtain and confirm data from investigators when needed.

\section{Methodological quality}

Various scales have been developed to assess methodological quality in an RCT, e.g. the Jadad criteria (Jadad et al. 1996). However, these scales are usually restricted to a few items rated as present, absent or unclear, leading to a small range of scores. Consequently, this makes it difficult to discern a relationship between methodological quality and effect size. In the present study, we used the Psychotherapy Outcome Study Methodology Rating Form by Öst (2008) which contains 22 items and has a total score ranging $0-44$. This instrument has previously been used in meta-analyses evaluating the efficacy of ACT (Öst, 2008, 2014) and cognitive behavioural treatments for OCD (Öst et al. $2015,2016)$. It is a valid measure of study quality as demonstrated by significant correlation with risk-of-bias ratings (Öst et al. 2015).

To assess the inter-rater reliability of the scale in the present review, the first author received $6 \mathrm{~h}$ of training in the use of the scale by the last author with various outcome studies as training examples. The first author then rated all the studies and a random selection of $20 \%$ of the studies was independently rated by the second author who had received the same amount of training in using the instrument. The intra-class correlation for the total score was 0.88 , and the kappa coefficients on the individual items varied between 0.84 and 0.97 , with a mean of 0.90 , indicating a high inter-rater reliability.

\section{Risk of bias ratings}

To assess possible risk of bias, each included study was also assessed using the criteria proposed by the Cochrane 
Table 1. Study inclusion and exclusion criteria

\begin{tabular}{|c|c|c|}
\hline & Inclusion criteria & Exclusion criteria $^{a}$ \\
\hline $\begin{array}{l}\text { Population and } \\
\text { conditions of } \\
\text { interest }\end{array}$ & $\begin{array}{l}\text { Paediatric and adult populations where participants } \\
\text { themselves applied for treatment; diagnosed with a } \\
\text { common psychiatric diagnosis; defined as any of the } \\
\text { following conditions: depression, recurrent depression, } \\
\text { anxiety disorder, insomnia, adjustment disorder, exhaustion } \\
\text { disorder/burnout syndrome. If a diagnostic procedure was } \\
\text { not used in the inclusion process, the participant had to } \\
\text { score above a target cutoff score }(M+2 \text { s.D.) on a validated } \\
\text { symptom scale targeting the disorder in question as } \\
\text { inclusion criterion }\end{array}$ & $\begin{array}{l}\text { Healthy individuals; participants with self-reported symptoms; } \\
\text { participants with previous depression who were currently in } \\
\text { remission, participants with alcohol- or substance use } \\
\text { disorders, severe psychiatric disorders (e.g., bipolar disorder, } \\
\text { psychosis or eating disorders) or neuropsychiatric diagnosis } \\
\text { (ADHD, autism, neurodevelopmental disorders); patients } \\
\text { with a somatic disorder/chronic illness and secondary } \\
\text { psychiatric symptoms; participants with caregiver stress; } \\
\text { studies were diagnosed participants were excluded; studies } \\
\text { were participants had not themselves applied for treatment }\end{array}$ \\
\hline Intervention & $\begin{array}{l}\text { Structured mindfulness meditation programs that followed } \\
\text { predetermined curricula (e.g., MBSR, MBCT and other MBIs). } \\
\text { That is, different types of MBIs were accepted, as long as the } \\
\text { main core of the treatment were attention training; } \\
\text { characterized by intentional and non-judgmental } \\
\text { observation of present moment experiences, including } \\
\text { bodily sensations, feelings, thoughts, and external stimuli } \\
\text { from the environment }\end{array}$ & $\begin{array}{l}\text { Studies where mindfulness meditation was not the foundation } \\
\text { and most of the intervention, including DBT and ACT; studies } \\
\text { where the mindfulness intervention was given remotely or } \\
\text { only by internet, video or audio (e.g., telephone or } \\
\text { smartphone) to an individual without the involvement of a } \\
\text { meditation teacher physically present; studies which also } \\
\text { included interventions above mindfulness (e.g., MBCT in } \\
\text { combination with a specified antidepressant medication) }\end{array}$ \\
\hline $\begin{array}{l}\text { Comparisons of } \\
\text { interest }\end{array}$ & $\begin{array}{l}\text { Both active and passive control conditions: Waitlist control; } \\
\text { no-treatment control; pill or psychological placebo } \\
\text { condition (pill or psychological placebo); } \\
\text { treatment-as-usual, other active psychological or } \\
\text { pharmacological treatment }\end{array}$ & None \\
\hline Outcome & $\begin{array}{l}\text { Symptoms of the psychiatric disorder (e.g., presence/absence } \\
\text { of clinical diagnosis, points on a validated symptom scale } \\
\text { for the disorder in question) }\end{array}$ & $\begin{array}{l}\text { General or global measures of psychological well-being (e.g., } \\
\text { measures of quality of life or mood), time to relapse in } \\
\text { depression }\end{array}$ \\
\hline Study design & RCTs with a control condition & Nonrandomized designs, such as observational studies \\
\hline Timing and setting & $\begin{array}{l}\text { Longitudinal studies that occurred in clinical settings or } \\
\text { community/university settings if they studied a clinical } \\
\text { population }\end{array}$ & $\begin{array}{l}\text { Community or university settings studying a healthy } \\
\text { population }\end{array}$ \\
\hline
\end{tabular}

$\mathrm{ACT}$, acceptance and commitment therapy; DBT, dialectical behavioural therapy; MBCT, mindfulness-based cognitive therapy; MBSR, mindfulness-based stress reduction; RCTs, randomized clinical trials.

${ }^{a}$ We excluded articles with no original data (reviews, editorials and comments), studies published in abstract form only, and dissertations.

collaboration (Higgins et al. 2011). Five dimensions were assessed: risk of selection bias due to the method of generating the randomization sequence, the risk of selection bias in terms of allocation concealment, detection bias in terms of blinding of outcome assessors, attrition bias due to incomplete outcome data and reporting bias due to selective reporting of results. Each dimension was rated as low risk, unclear or high risk, where the alternative 'unclear' was used only when there was no data to assess the quality criterion in the original study. The scoring was 0 for low risk, 0.5 for unclear, and 1 for high risk, yielding a range of $0-5$ for the risk of bias ratings. For studies using self-report measures, the criterion of blinding of outcome assessors was judged as high risk. Risk of bias ratings was analysed as a potential moderator of treatment effect.

\section{Assessment of evidence status}

To assess the evidence status of MBIs for the respective psychiatric disorders, we applied the evidence criteria for empirically supported treatments (ESTs) developed by the American Psychological Association (APA) Division 12 (Chambless et al. 1998) using the most recent revision (Southam-Gerow \& Prinstein, 2014). The rationale for using the APA criteria rather than any other system (e.g., Balshem et al. 2011), in assessing the evidence is that the former is developed especially for evaluating psychological treatments, and contains distinct rating criteria which minimize a subjective evaluation of the evidence. To summarize this process for evaluating evidence; each study is first assessed regarding five different methodological criteria (study design, the definition of an independent variable, clear description of the population, outcome measures and analysis adequacy). The treatment is thereafter classified into one out of four categories with respect to evidence status. For each category or level of evidence, specific criteria are used (for a full description of the criteria, see Southam-Gerow \& Prinstein, 2014). Treatments can be defined as being well-established (indicating strong research support), probably efficacious (indicating modest research support), possibly efficacious (indicating weak research support), experimental (indicating no research support) or of controversial efficacy. For a treatment to be judged as well-established, probably efficacious or possibly efficacious, the classification must be based on original studies fulfilling all five methodological criteria.

\section{Statistical analyses}

All statistical analyses were conducted using Comprehensive MetaAnalysis software (CMA, version 3 for Windows). For each study, the main outcome measure assessing symptom reduction of the targeted disorder was extracted. Effect sizes were calculated as Hedges' $g$; that is the difference in means between the MBI- and control condition was divided by the pooled s.D., corrected for small sample size, separately for post- and follow-up assessments (Hedges, 1982). 


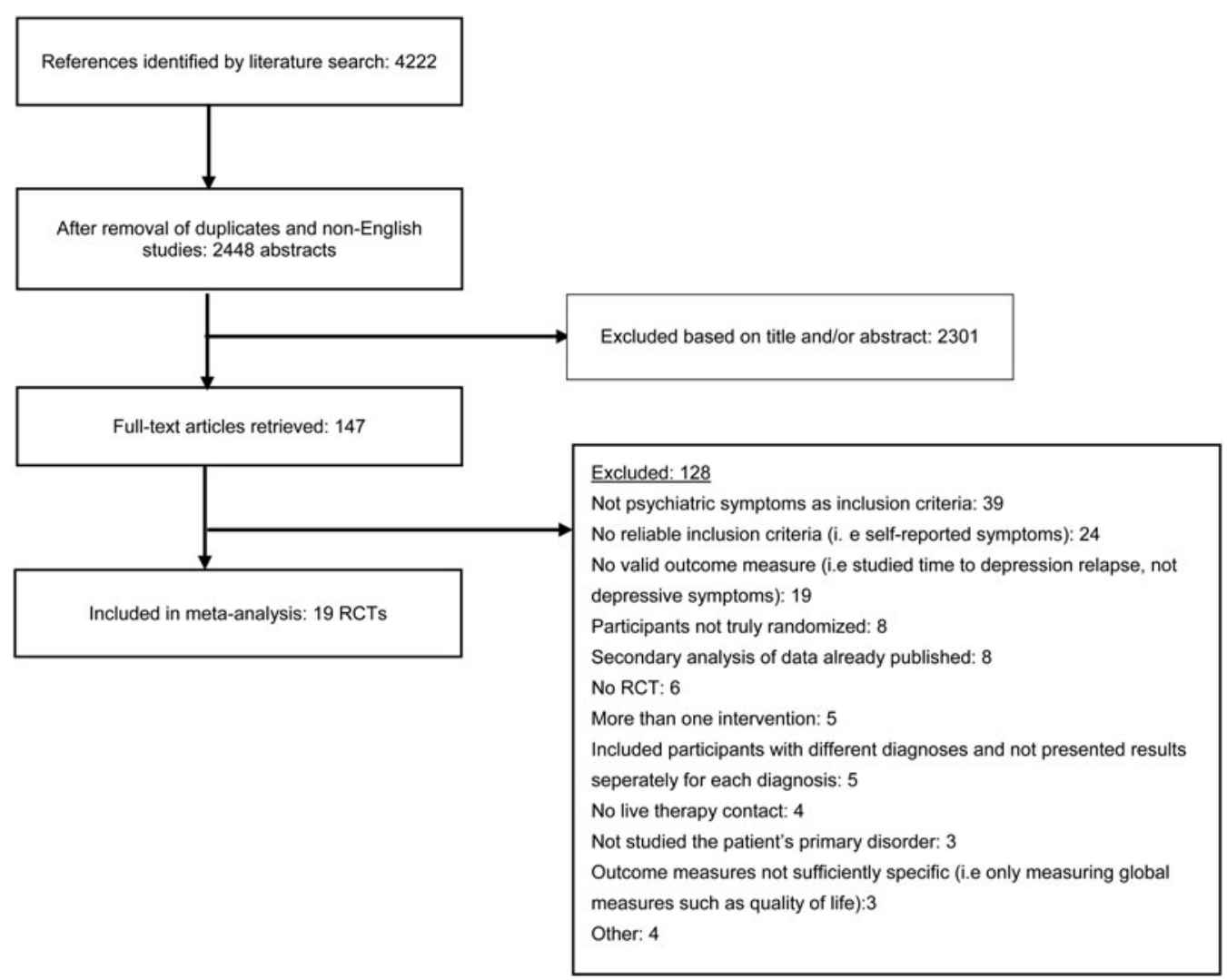

Fig. 1. Flowchart of inclusion of studies.

Before pooling effect sizes we screened for statistical outliers, which was defined as being outside $M+2$ s.D. Outliers (2.2\%) were then reduced to the exact value of $M+2$ S.D. by winsorising (Lipsey \& Wilson, 2001). In the between-group effects analysis, which was the main analysis, we used scores on the primary outcome measure in the MBI condition compared with the control condition(s). Within-group effects were calculated using pre- and post- or follow-up data for the MBI condition and all comparison conditions. Assessment of heterogeneity was calculated using $I^{2}$, which is the proportion of variance due to true heterogeneity, and Cochran's $Q$, which tests if that heterogeneity is statistically significant. Publication bias was assessed using Egger's regression intercept (Egger et al. 1997) and Duval \& Tweedie's trim-and-fill-method (Duval \& Tweedie, 2000). Subgroup- and meta-regression analyses were conducted to assess whether different category- and continuous variables were related to the effect size. To ensure the validity of the subgroup- and meta-regression analyses, they were conducted only on variables in which $\geqslant 75 \%$ of the studies had available data. The full list of pre-specified moderator variables is presented in online Table OSM2 in the OSM.

\section{Results}

\section{Systematic review}

\section{Included studies}

The literature search resulted in a total of 4222 articles, which after excluding duplicates and non-English articles was reduced to 2448 articles. Titles and abstracts of these studies were screened, and after removal of 2301 irrelevant studies, 147 full-text articles were retrieved. These were reviewed in detail, and 128 were excluded as they did not meet eligibility criteria. A total of 19 studies were thus included in the systematic review and meta-analysis. Figure 1 shows a flowchart of the inclusion of studies in the current meta-analysis, with details for the exclusion of trials. All excluded studies with the reason for exclusion are found in the online Appendix.

\section{Characteristics of included studies}

The characteristics of the included RCTs regarding background and intervention characteristics are summarized in Tables 2 and 3 , respectively. The total number of participants in the 19 included studies were $N=1291$, with the sample size varying from 26 to 116 . The proportion of participants declining participation in the study, either not giving informed consent after being offered to participate, or doing so but not showing up for the first session, was reported in all but one study and varied from 0 to $43.6 \%$ with a mean of $16.7 \%$. The attrition rate (participating in at least the first session but stopping before completing the agreed-upon number of sessions) was reported in 17 of 19 studies, and varied between 1.4 and $23.3 \%$, with a mean of $12.1 \%$. The disorders under study were depression $(n=5)$, post-traumatic stress disorder (PTSD) $(n=4)$, social anxiety disorder $(n=3)$, generalized anxiety disorder $(n=2)$, insomnia $(n=2)$, hypochondriasis $(n=1)$ and elevated stress $(n=1)$. Mean participant age ranged from 21.8 (s.D. = 2.69 ) to 78.1 (S.D. $=2.98$ ) with an average of 44.7 , and the average proportion of women were $50 \%$. The evaluated MBIs included MBSR, MBCT, mindful awareness practice (MAP), brief mindfulness training (BMT) and body-scan mindfulness meditation (MM). Comparison conditions included no-treatment control, waitlist control, treatment-as-usual (TAU), placebo, antidepressant 
Table 2. Background characteristics of included studies

\begin{tabular}{|c|c|c|c|c|c|c|c|c|c|c|c|c|}
\hline Disorder & Study & MBI type & Country & Comparison $^{a}$ & $\stackrel{N}{N}$ & $\begin{array}{c}\% \\
\text { females }\end{array}$ & $\begin{array}{l}\text { Age } \\
\text { (M) }\end{array}$ & $\begin{array}{c}\% \\
\text { decliners }\end{array}$ & $\begin{array}{l}N \text { included } \\
\text { rand. to } T x\end{array}$ & $\begin{array}{l}N \text { included } \\
\text { rand. to } \\
\text { control }\end{array}$ & $\begin{array}{c}\% \\
\text { comorbidity }^{b}\end{array}$ & $\begin{array}{c}\% \text { current } \\
\text { drug Tx }\end{array}$ \\
\hline GAD & Asmaee Majid et al. (2012) & MBSR & Iran & NTC & 33 & 0 & 32.2 & 5.7 & 16 & 15 & NR & NR \\
\hline Depression & Barnhofer et al. (2009) & МВCT & UK & TAU & 31 & 67.9 & 41.2 & 43.6 & 16 & 15 & 42.9 & 60.7 \\
\hline Depression & Chiesa et al. (2015) & $\mathrm{MBCT}$ & Italy & PLA & 43 & 72.1 & 49 & 14 & 23 & 20 & NR & 100 \\
\hline Depression & Eisendrath et al. (2016) & $\mathrm{MBCT}+\mathrm{TAU}$ & USA & PLA & 173 & 76.3 & 46.2 & 8.5 & 87 & 86 & 60 & 100 \\
\hline Stress & Guardino et al. (2014) & MAP & USA & PLA & 47 & 100 & 33.1 & 6 & 24 & 23 & 31 & NR \\
\hline Dysthymia & Hamidian et al. (2013) & $\mathrm{MBCT}+\mathrm{TAU}$ & Iran & ADM & 50 & NR & NR & NR & 25 & 25 & NR & NR \\
\hline GAD & Hoge et al. (2013) & MBSR & USA & PLA & 89 & 50.6 & 39.2 & 27 & 48 & 41 & 28.1 & 19.1 \\
\hline SAD & Jazaieri et al. (2012) & MBSR & USA & Exercise & 56 & 35.7 & 32.9 & 29.9 & 29 & 25 & 32.1 & 0 \\
\hline PTSD & Kearney et al. (2013) & $M B S R+T A U$ & USA & TAU & 47 & 21.3 & 52 & 0 & 25 & 22 & NR & 64 \\
\hline SAD & Koszycki et al. (2007) & MBSR & Canada & CBT & 53 & 52.8 & 38.2 & 11.3 & 26 & 27 & 18.9 & 28.3 \\
\hline Hypochondriasis & McManus et al. (2012) & MBCT & UK & TAU & 74 & 78.4 & 42.6 & 11.1 & 36 & 38 & 38.7 & 41.9 \\
\hline Depression & Michalak et al. (2015) & MBCT + TAU & Germany & $\mathrm{CT}+\mathrm{TAU}$ & 106 & 62.3 & 50.8 & 38.4 & 36 & 35 & 42.2 & 53.8 \\
\hline Insomnia & Ong et al. (2014) & MBSR & USA & MBCBT & 54 & 74.1 & 42.9 & 7.4 & 19 & 19 & NR & NR \\
\hline SAD & Piet et al. (2010) & МBCT & Denmark & CBT & 26 & 69.2 & 21.8 & 21.2 & 14 & 12 & 42.3 & 0 \\
\hline PTSD & Polusny et al. (2015) & MBSR & USA & PLA & 116 & 16 & 58.5 & 1.7 & 58 & 58 & 42.2 & 86 \\
\hline PTSD & Possemato et al. (2015) & $\mathrm{BMT}+\mathrm{TAU}$ & USA & TAU & 62 & 12.9 & 46.4 & 28.1 & 36 & 26 & NR & NR \\
\hline Depression & van Aalderen et al. (2012) & МBCT & Holland & TAU & 69 & 65.2 & 47.5 & 2.8 & 34 & 35 & NR & 49.3 \\
\hline PTSD & Wahbeh et al. (2016) & MM & USA & Biofeedback & 102 & 5.9 & 52.1 & 10.5 & 27 & 25 & NR & NR \\
\hline Insomnia & Zhang et al. (2015) & MBSR & China & WLC & 60 & 41.7 & 78.1 & 33.3 & 30 & 30 & 0 & NR \\
\hline
\end{tabular}

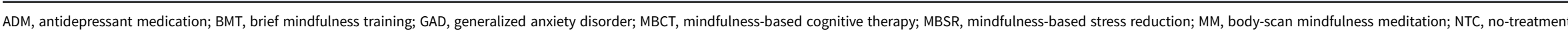
control; PLA, placebo; PTSD, post-traumatic stress disorder; SAD, social anxiety disorder; TAU, treatment as usual; NR, not reported; WLC, waiting-list control; Tx, treatment.

Main comparison if more than one comparison group. 
Table 3. Intervention characteristics of included studies

\begin{tabular}{|c|c|c|c|c|c|c|c|c|c|c|}
\hline Study & $\begin{array}{c}\text { MBI } \\
\text { subtype }\end{array}$ & Therapist profession & $\begin{array}{l}\text { Therapist } \\
\text { qualification }\end{array}$ & $\begin{array}{c}\text { Tx } \\
\text { format }\end{array}$ & $\begin{array}{l}\text { Attrition } \\
(\%)\end{array}$ & $\begin{array}{c}\text { Tx } \\
\text { duration }\end{array}$ & $\begin{array}{l}\text { No. of } \\
\text { sessions }\end{array}$ & $\begin{array}{l}\text { Total Tx } \\
\text { time (h) }\end{array}$ & $\begin{array}{c}\mathrm{Tx} \\
\text { intensity }\end{array}$ & $\begin{array}{l}\text { F-up } \\
\text { months }\end{array}$ \\
\hline Asmaee Majid et al. (2012) & MBSR & NR & 3 & G & NR & 8 & 8 & 16 & 2 & 0 \\
\hline Barnhofer et al. (2009) & MBCT & Fully qualified CBT therapist & 1 & G & 9.7 & 8 & 8 & 16 & 2 & 0 \\
\hline Chiesa et al. (2015) & $\mathrm{MBCT}$ & Psychiatrist, psychotherapist & 3 & G & 23.3 & 8 & 8 & 16 & 2 & 4.5 \\
\hline Eisendrath et al. (2016) & $\mathrm{MBCT}+\mathrm{TAU}$ & NR & 2 & G & 14.5 & 8 & 8 & 18 & 2.25 & 0 \\
\hline Guardino et al. (2014) & MAP & NR & 3 & G & 8.5 & 6 & 6 & 12 & 2 & 1.5 \\
\hline Hamidian et al. (2013) & $\mathrm{MBCT}+\mathrm{TAU}$ & NR & 0 & G & 12 & 8 & 8 & 20 & 2.5 & 0 \\
\hline Hoge et al. (2013) & MBSR & NR & 3 & G & 11.2 & 8 & 9 & 20 & 2.5 & 0 \\
\hline Jazaieri et al. (2012) & MBSR & NR & 3 & G & 8.9 & 8 & 9 & 27.5 & 3.25 & 3 \\
\hline Kearney et al. (2013) & MBSR + TAU & NR & 3 & G & 6.3 & 8 & 9 & 27 & 3.38 & 4 \\
\hline Koszycki et al. (2007) & MBSR & Other/no mental health professional & 3 & G & 22.6 & 8 & 9 & 27.5 & 3.44 & 0 \\
\hline McManus et al. (2012) & MBCT & NR & 3 & G & 1.4 & 8 & 8 & 16 & 2 & 12 \\
\hline Michalak et al. (2015) & $\mathrm{MBCT}+\mathrm{TAU}$ & $\begin{array}{l}\text { Psychologist, Psychiatrist, } \\
\text { psychologist + psychotherapist }\end{array}$ & 3 & G & 18.9 & 8 & 8 & 20 & 2.5 & 0 \\
\hline Ong et al. (2014) & MBSR & Psychologist, physician & 3 & G & 11.1 & 8 & 9 & 26 & 3.25 & 6 \\
\hline Piet et al. (2010) & MBCT & NR & 3 & G & 15.4 & 8 & 8 & 16 & 2 & 12 \\
\hline Polusny et al. (2015) & MBSR & Psychologists & 1 & G & 14.7 & 8 & 9 & 26.5 & 3.31 & 2 \\
\hline Possemato et al. (2015) & $\mathrm{BMT}+\mathrm{TAU}$ & Physician & 3 & G & 9.7 & 4 & 4 & 6 & 1.5 & 1 \\
\hline van Aalderen et al. (2012) & MBCT & $\begin{array}{l}\text { Psychiatrist + CBT therapist, psychologist, } \\
\text { occupational therapist }\end{array}$ & 3 & G & 13 & 8 & 9 & 26 & 3.25 & 12 \\
\hline Wahbeh et al. (2016) & MM & NR & 1 & 1 & NR & 6 & 6 & 6 & 1 & 0 \\
\hline Zhang et al. (2015) & MBSR & NR & 3 & G & 5 & 8 & 9 & 18 & 2.25 & 0 \\
\hline
\end{tabular}

BMT, brief mindfulness training; MBCT, mindfulness-based cognitive therapy; MBSR, mindfulness-based stress reduction; MM, body-scan mindfulness meditation; TAU, treatment as usual; NR, not reported; Tx, treatment; G, group; I, individual.

Categories as follows: $0=$ not reported, $1=$ training in the treatment, $2=$ experience in delivering the treatment, $3=$ instructor/teacher in the method. 
medication, cognitive behaviour therapy (CBT), mindfulness-based CBT (a combination of mindfulness and CBT components), biofeedback and aerobic exercise. The number of comparison conditions within each study ranged from one to three.

\section{Methodological quality}

The average score on the scale of methodological quality (Öst, 2008) was 19.16 (S.D. $=5.18$ ), ranging from 10 to 28 . The items yielding the lowest scores were 'Checks for treatment adherence' (only four studies reported acceptable data, i.e. one out of maximum two points); and 'Checks for therapist competence' (only three studies reported acceptable data). Moreover, blinded assessors of outcome measures were used in nine of 19 studies, but none of these studies reported testing of blindness. The majority of the studies included (11 of 19) had only one therapist meaning that there was a complete confounding of the therapist and treatment effects. None of the remaining eight studies which had more than one therapist analysed the therapist factor as a moderator of effect. In sum, results of ratings of methodological study quality suggest that the internal validity was low in many of the included RCTs.

\section{Risk of bias}

Only two studies (Barnhofer et al. 2009; Chiesa et al. 2015) were judged as having a low risk of bias on all dimensions on the Cochrane Risk of Bias criteria. Eight studies (Piet et al. 2010; Asmaee Majid et al. 2012; Jazaieri et al. 2012; Kearney et al. 2013; Guardino et al. 2014; Ong et al. 2014; Zhang et al. 2015; Wahbeh et al. 2016) out of 19 had high risk of detection bias and only five studies (Barnhofer et al. 2009; Kearney et al. 2013; Ong et al. 2014; Chiesa et al. 2015; Michalak et al. 2015) had low risk of selection bias in terms of allocation concealment. The lowest risk of bias was found on the reporting bias dimension, where only three studies had high $(n=2)$ or unclear $(n=1)$ risk of bias. See online Table OSM11 in the OSM for results on risk of bias in each study and online Figure OSM2 for the averaged risk of bias in the included studies.

\section{Meta-analysis results}

\section{Attrition}

The dropout rate for the different treatment conditions ranged between $2.2 \%$ for the waitlist conditions and $9.2 \%$ for the placebo groups, with a dropout rate for MBI groups of 7.8\% [95\% confidence interval (CI 5.7-10.7)]. A subgroup analysis showed that the difference in attrition between study conditions was nonsignificant $\left[Q_{\text {between }}(4)=3.71, p=0.45\right]$. See online Table OSM4 in the OSM for a description of dropout rate for the different treatment conditions in the included studies.

\section{Between-group comparisons of treatment effect}

Figure 2 displays the forest plot of the standardized betweengroup effect sizes (Hedges' $g$ ) of MBI studies. Results of analyses at post-treatment are presented in Table 4 with effect sizes, 95\% CIs and heterogeneity estimates. As can be seen from Table 4, the effect size across all comparison conditions was small and significant. MBIs were significantly more effective than waitlist control, but not more effective than placebo or other active treatments. The effect size was large compared with waiting-list control and small for all other comparisons. Seven studies reported data at follow-up (ranging from 1.5 to 12 months), with an overall effect size (MBIs $v$. all comparisons; which included placebo, TAU and other treatments) of $g=0.16$. A full description of results of follow-up analyses is presented in online Table OSM5 in the OSM. For a description of post-treatment

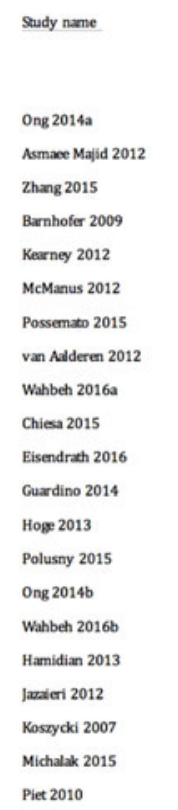

Comparison
WCC
W.C
WLC
TAU
TAU
TAU
TAU
TAU
PLA
PLA
PLA
PLA
PLA
PLA
Other
Other
Other
Other
CBT
CBT
CBT

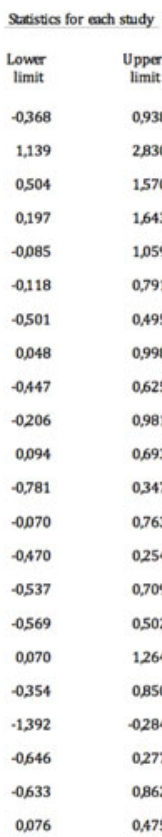

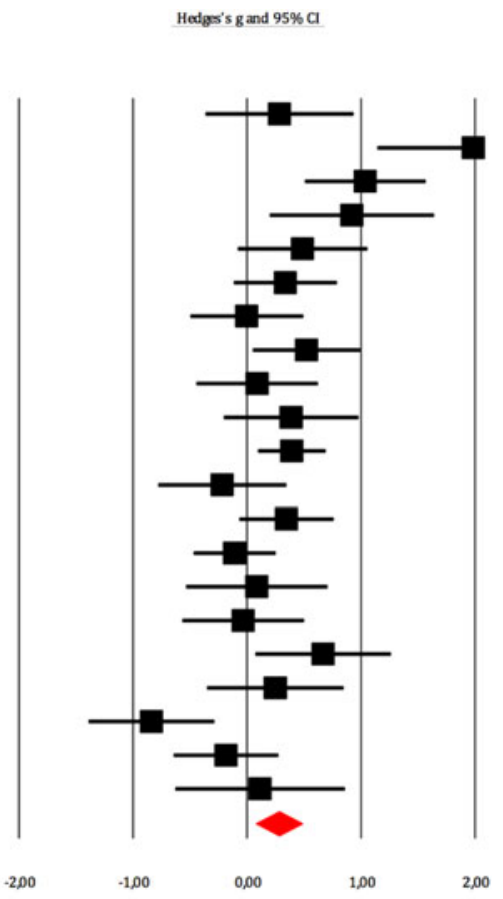

Favours comp. Favours MBI

Fig. 2. Between-group effect sizes (Hedges' g). 
Table 4. Effect sizes (Hedges' $g$ ) on the primary outcome measure for all MBIs RCTs and divided on comparison conditions at post-treatment assessments

\begin{tabular}{|c|c|c|c|c|c|c|}
\hline Comparison & $k$ & $g$-value & $95 \% \mathrm{Cl}$ & $z$-value & Q-value & $I^{2}$ \\
\hline All studies & 23 & 0.28 & $0.08,0.48$ & $2.78^{\mathrm{b}}$ & $66.7^{c}$ & 67 \\
\hline $\mathrm{MBI} v . \mathrm{WLC}+\mathrm{NTC}$ & 3 & 1.07 & $0.21,1.92$ & $2.45^{\mathrm{a}}$ & $9.8^{b}$ & 80 \\
\hline MBI v. Placebo & 6 & 0.17 & $-0.05,0.39$ & -1.52 & 7.5 & 33 \\
\hline MBI v. TAU & 6 & 0.40 & $0.19,0.61$ & $3.74^{c}$ & 4.9 & 0 \\
\hline MBI $v$. active $T x$ & 8 & -0.01 & $-0.35,0.33$ & -0.06 & $19.7^{\mathrm{b}}$ & 64 \\
\hline MBI v. CBT & 3 & -0.33 & $-0.86,0.19$ & -1.24 & 4.9 & 60 \\
\hline MBI $v$. other Tx & 5 & 0.18 & $-0.20,0.56$ & 0.92 & 8.6 & 54 \\
\hline
\end{tabular}

$k$, number of comparisons; WLC, waitlist control; NTC, no treatment control; TAU, treatment-as-usual; Tx, treatment. A positive $g$-value means that the first treatment in the comparison is more effective and a negative that the second is more effective.

${ }^{\mathrm{a}} p<0.05,{ }^{\mathrm{b}} p<0.01,{ }^{\mathrm{c}} p<0.001$.

effect sizes stratified by psychiatric disorder, see Table 5. As shown in Table 5, MBIs showed a significant between-group effect at post-treatment in the treatment of depression $(g=0.40)$, but not for any of the other disorders.

\section{Moderator analyses}

Meta-regression analyses of continuous variables showed two significant moderators of the effect size. Methodological quality was negatively related to outcome $(z=-2.51, p=0.012)$, i.e. the lower methodological quality of the study the larger the effect. Treatment duration was positively related to outcome $(z=2.33$, $p=0.020$ ), i.e., more treatment weeks yielded a higher treatment effect. No other continuous variables (i.e., number of patients, mean age, per cent females, the risk of bias, per cent decliners, number of sessions, total hours of treatment, treatment intensity or attrition) were significant moderators. Subgroup analyses did not result in any significant category variables moderating the outcome. See online Tables OSM6 and OSM7 in the OSM for complete results of meta-regression and subgroups analyses respectively, including comparisons between disorders.

\section{Within-group effect sizes}

Within-group effect sizes for MBIs and all control conditions are summarized in online Table OSM7 in the OSM. A significant effect of a moderate magnitude was found for all included comparisons $(g=0.69)$. For MBIs, the effect size at post-treatment was large $(g=0.98)$. Placebo $(g=0.61)$, TAU $(g=0.27)$ and other active treatments $(g=0.74)$, with a subgroup of cognitive behavioural treatments $(g=1.20)$, also showed significant effect sizes ranging from small (TAU) to large (CBT) at post-treatment. Results at follow-up indicated a lasting effect for all control conditions but not for MBIs, where a small deterioration was observed $(g=0.87)$. Due to the low proportion of studies contributing with follow-up data, we recalculated the post-treatment within-group effect including only the studies that contributed with both post- and follow-up data, to get a more valid test of the long-term effects. The new modified estimated within-group effect sizes for MBIs were $g=0.77(z=8.57, p<0.0001)$ at posttreatment and $g=0.87(z=7.00, p<0.001)$ at follow-up, thus indicating a small improvement.

\section{Publication bias}

Publication bias analysis using Egger's regression intercept method (Egger et al. 1997) was not significant $(t=0.95)$, but Duval \& Tweedie's (2000) trim-and-fill method suggested four trimmed studies. Results from publication bias analysis and a funnel plot for MBIs $v$. all comparisons are found in online Table OSM8 and Figure OSM3 in the OSM.

\section{Evaluation of the evidence base of MBIs}

The RCTs on MBIs for the different psychiatric disorders are summarized in relation to the criteria for ESTs in Table 6. For one disorder, hypochondriasis (health anxiety), for which there was only one study (McManus et al. 2012), the MBI was judged to be possibly efficacious (level 3, corresponding to weak research support). This was because that study fulfilled all five of the method criteria and demonstrated MBI to be superior to treatmentas-usual. For all other psychiatric disorders evaluated in this meta-analysis (depression, PTSD, generalized anxiety disorder, social anxiety disorder, elevated stress and insomnia) no study fulfilled all methodological criteria and the research support for MBIs must, therefore, be judged as experimental (level 4; corresponding to no research support) for these disorders. The most common shortcomings in the studies were lack of clear description of the population (criterion 3) and lack of reliable and valid outcome measures (criterion 4).

\section{Discussion}

The aims of this systematic review and meta-analysis were: (1) to quantify the size of effect of MBIs for common psychiatric disorders in the acute phase; (2) to explore moderator variables contributing to the treatment effect; and (3) to evaluate the evidence status of MBIs for the psychiatric disorders it has been tried for. The results showed that the average effect size for MBIs across all comparison conditions was small $(g=0.28)$ and that it was only significantly superior to non-active control conditions. Moderator analyses showed that lower study quality and longer treatment duration were related to larger effect sizes. Furthermore, subgroup analyses revealed that depression was the only disorder for which the effects were significant. Assessment of the evidence status showed that MBIs are to be considered experimental for all included psychiatric populations except for hypochondriasis (health anxiety), for which MBI was classified as a possibly efficacious treatment. The evidence base for MBIs can be concluded as weak, and MBIs can, thus, not yet be regarded as an evidence-based treatment for common psychiatric disorders. 
Table 5. Subgroup analysis of treatment effect at post-treatment

\begin{tabular}{|c|c|c|c|c|c|}
\hline Variable & $k$ & $g$-value & $95 \% \mathrm{Cl}$ & $Q_{b}$-value & $p$ value \\
\hline Type of comparison & & & & 2.672 & 0.102 \\
\hline Active treatment & 20 & 0.18 & $0.01,0.35$ & & \\
\hline Passive control & 3 & 1.00 & $0.03,1.97$ & & \\
\hline Type of data analysis & & & & 0.493 & 0.483 \\
\hline Completer & 6 & 0.44 & $-0.11,0.98$ & & \\
\hline Intent-to-treat & 17 & 0.23 & $0.03,0.42$ & & \\
\hline Treatment format & & & & 1.257 & 0.262 \\
\hline Group & 20 & 0.31 & $0.11,0.52$ & & \\
\hline Individual & 3 & 0.01 & $-0.49,0.50$ & & \\
\hline Disorder & & & & 9.877 & 0.130 \\
\hline Depression & 7 & 0.40 & $0.17,0.63$ & & \\
\hline GAD & 2 & 1.12 & $-0.49,2.73$ & & \\
\hline Hypochondriasis & 1 & 0.34 & $-0.12,0.79$ & & \\
\hline Insomnia & 3 & 0.49 & $-0.11,1.09$ & & \\
\hline PTSD & 6 & 0.05 & $-0.21,0.30$ & & \\
\hline SAD & 3 & -0.18 & $-0.90,0.54$ & & \\
\hline Stress & 1 & -0.22 & $-0.78,0.35$ & & \\
\hline Inclusion criteria & & & & 4.315 & 0.229 \\
\hline Cutoff score & 1 & -0.21 & $-0.78,0.35$ & & \\
\hline Diagnosis & 16 & 0.35 & $0.13,0.57$ & & \\
\hline Diagnosis + cut-off score & 4 & -0.01 & $-0.58,0.57$ & & \\
\hline Diagnosis or symptom level & 2 & 0.42 & $-0.48,1.32$ & & \\
\hline Therapist qualifications & & & & 2.622 & 0.454 \\
\hline Not reported & 1 & 0.67 & $0.07,1.26$ & & \\
\hline Trained & 5 & 0.12 & $-0.28,0.51$ & & \\
\hline Experienced & 1 & 0.39 & $0.09,0.69$ & & \\
\hline Instructor in the method & 16 & 0.29 & $0.04,0.54$ & & \\
\hline Country & & & & 2.285 & 0.131 \\
\hline USA & 12 & 0.14 & $-0.02 ; 0.30$ & & \\
\hline Other & 11 & 0.45 & $0.08 ; 0.82$ & & \\
\hline
\end{tabular}

k, number of comparisons; $Q_{b}, Q$ between subgroups; GAD, generalized anxiety disorder; PTSD, post-traumatic stress disorder; SAD, social anxiety disorder.

Regarding the first aim, our meta-analysis showed MBIs to be superior to no treatment, waitlist control or treatment-as-usual, but not significantly more effective than placebo or other active treatments. The observed effect size was negative $(g=-0.33)$ for MBIs when compared with CBT, which is the psychological treatment with strongest empirical support for common psychiatric disorders (National Institute for Clinical Excellence, 2011; American Psychological Association, 2016). However, this effect was not significant and the wide CI indicates the low precision of the effect estimate. This means that although it cannot be concluded that MBIs is inferior to CBT, at the same time one cannot regard MBIs as non-inferior to $\mathrm{CBT}$; i.e. there is no support for the notion that MBIs yields equal or comparable treatment effect as CBT for common psychiatric disorders. This stands in contrasts to some clinical studies, e.g. a recent study by Sundqvist et al. (2015), which compared mindfulness group therapy to treatment-as-usual (where $76 \%$ in the latter group received individual CBT, on average six sessions) in a primary care context.

One aspect that warrants commenting is whether our approach to aggregating effects for different MBIs across several CMDs confers the risk of diluting effects for highly effective specific MBIs for specific CMDs. The methodology of aggregating across disorders has previously been used in meta-analyses both for MBIs (e.g., Hofmann et al. 2010 and Khoury et al. 2013) and for CBT (i.e., Shapiro \& Shapiro, 1982; Wampold et al. 1997 and Weisz et al., 1995). To provide a full picture we have also reported effect sizes for each specific disorder (Table 5), and it should be noted that the well-researched MBCT and MBSR were the clearly most common forms of MBIs. It should also be acknowledged that there was some variation in observed effect sizes between disorders. The only disorder where there was a significant effect of MBIs was depression, which might suggest that MBIs could be 
Table 6 Summary of mindfulness-based intervention (MBI) studies for psychiatric disorders in relation to APA criteria for empirically supported treatments (ESTs).

\begin{tabular}{|c|c|c|c|c|c|c|c|c|c|}
\hline Study & $\begin{array}{l}\text { Comparison } \\
\text { condition }\end{array}$ & WLC & Placebo & TAU & $\begin{array}{l}\text { Established } \\
\text { treatment }\end{array}$ & $\begin{array}{l}\text { Treatment } \\
\text { manuals }\end{array}$ & $\begin{array}{l}\text { Inclusion criteria } \\
\text { reliably delineated }\end{array}$ & $\begin{array}{l}\text { Reliable and valid } \\
\text { outcome measures }\end{array}$ & $\begin{array}{l}\text { Appropriate } \\
\text { data analysis }\end{array}$ \\
\hline \multicolumn{10}{|l|}{ Depression } \\
\hline Barnhofer 2009 & TAU & & & $>$ & & + & - & - & + \\
\hline Chiesa 2015 & PLA & & $>$ & & & + & - & + & + \\
\hline Eisendrath 2016 & PLA & & $>$ & & & + & - & - & $?$ \\
\hline Michalak 2015 & CT & & & & $=$ & + & - & - & + \\
\hline Michalak 2015 & TAU & & & $=$ & & + & - & - & + \\
\hline van Aalderen 2012 & TAU & & & $>$ & & + & - & + & + \\
\hline Hamidian 2013 & ADM & & & & $>$ & + & - & - & $?$ \\
\hline \multicolumn{10}{|l|}{ PTSD } \\
\hline Kearney 2012 & TAU & & & $=$ & & + & - & + & + \\
\hline Polusny 2015 & PLA & & $>$ & & & + & - & + & + \\
\hline Possemato 2015 & TAU & & & $=$ & & + & - & + & + \\
\hline Wahbeh 2016 & Biofeedback & & & & $(=)$ & $?$ & - & + & $?$ \\
\hline Wahbeh 2016 & PLA & & $=$ & & & $?$ & - & + & $?$ \\
\hline Wahbeh 2016 & MBI + Biofeedback & & & & $\Leftrightarrow$ & $?$ & - & + & $?$ \\
\hline \multicolumn{10}{|c|}{ Generalized anxiety disorder } \\
\hline Asmaee Majid 2012 & NTC & $>$ & & & & $?$ & - & + & $?$ \\
\hline Hoge 2013 & PLA & & $=$ & & & + & - & - & + \\
\hline \multicolumn{10}{|l|}{$\begin{array}{l}\text { Social Anxiety } \\
\text { Disorder }\end{array}$} \\
\hline Jazaieri 2012 & Exercise & & & & $(=)$ & + & - & + & $?$ \\
\hline Koszycki 2007 & CBT & & & & $<$ & + & - & + & + \\
\hline Piet 2010 & CBT & & & & $=$ & + & - & - & + \\
\hline \multicolumn{10}{|l|}{ Hypochondriasis } \\
\hline McManus 2012 & TAU & & & $>$ & & + & + & + & + \\
\hline \multicolumn{10}{|l|}{ Stress \& anxiety } \\
\hline Guardino 2014 & PLA & & $>$ & & & + & - & - & + \\
\hline \multicolumn{10}{|l|}{ Chronic insomnia } \\
\hline Ong 2014 & MBCBT & & & & $(=)$ & + & - & - & + \\
\hline Ong 2014 & WLC & $>$ & & & & + & - & - & + \\
\hline Zhang 2015 & WLC & $>$ & & & & - & - & - & + \\
\hline
\end{tabular}

Note: $A D M=$ Antidepressant medication, $C T=$ cognitive therapy, MBCBT = Mindfulness-based CBT for insomnia, NTC = No-treatment control, PLA = placebo, TAU = treatment as usual, WLC = waitlist control. $>=$ significantly more effective than the

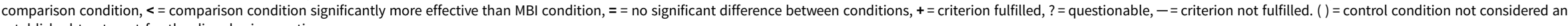
established treatment for the disorder in question. 
more suitable for depression than the other disorders investigated in the present meta-analysis. However, as there were so few studies conducted for most specific disorders (three or fewer RCTs for all conditions except depression and PTSD), the non-significant effect sizes for most conditions is probably also related to low power, and as can be read from Table 5, there was considerable uncertainty around the effect estimates.

Concerning the second aim of the study, moderator analyses revealed that study quality and treatment length were significant moderators of treatment effect. The finding that methodological quality is a negative predictor of effect means that studies of lower quality tended to show larger effect sizes. It is therefore likely that the overall effect size observed for MBIs against all comparison conditions $(g=0.28)$ is somewhat overestimated because this estimate includes studies of low methodological quality. Moreover, this has important implications when assessing the evidence status for any given treatment: if a large proportion of the studies are of low methodological quality, the results may not be a valid indicator of whether the treatment is effective as systematic bias may contribute to the observed effect. For a treatment to be regarded as wellestablished one must also show that it is more effective than placebo, or equally effective to another well-established treatment. Only demonstrating superiority to waitlist, no-treatment or treatment-as-usual is not sufficient. When it comes to the other significant moderator of treatment effect, i.e. that treatment length was a positive predictor, it should be noted that the available data did not allow us to draw any conclusions regarding the optimal number of sessions. Out of the 19 studies included in the meta-analysis, 16 (all of them MBSR or MBCT programs) used eight or nine sessions. Hence, there is a restriction of range making an estimation of dose-response relationship uninformative. However, it can be of interest to note that when excluding the studies with $<8$ sessions the overall ES was $g=0.35$ ( $p=0.003$ ), as compared with $g=0.28(p=0.007)$ when including all studies.

The third aim of the study was to evaluate MBIs against criteria for evidence-based treatment for the disorders for which it has been tested. Results show that for all but one disorder, MBIs can be regarded as an experimental treatment, i.e. there is no research support for the efficacy of MBIs for the psychiatric disorders evaluated. The exception was hypochondriasis, where the research support was judged as possibly efficacious. The evaluation of MBIs as lacking empirical support is generally due to methodological shortcomings in the existing studies. Important and commonly occurring limitations noted in this meta-analysis were: (a) low reliability in procedures for assessing psychiatric diagnoses, i.e. reliable diagnostic instruments were often not used and inter-rater-reliability estimates not reported; (b) lack of checks for treatment adherence and therapist competence; (c) lack of blinded outcome assessors; and (d) the use of only one therapist.

\section{Results in relation to earlier meta-analyses and RCTS}

Compared with previously published meta-analyses and reviews evaluating MBIs, the results from the current meta-analysis are on the conservative side; contrasting several of those who have found MBIs to be a promising treatment with increasing research support (e.g., Khoury et al. 2013 and Demarzo et al. 2015b).

One possible explanation for this is that the present metaanalysis differs from the previous ones (with the exception of Strauss et al. 2014), in that we only included RCTs investigating populations with a manifest psychiatric disorder as a principal diagnosis. This was because our aim was to assess the effects and the evidence of MBIs in the treatment of common psychiatric disorders in the acute phase. A consequence of using this perspective was also that the outcome measures used in our analyses differed somewhat from those in many previously published trials of MBIs where generic measures, such as quality of life, often have been used. We, however, regarded it to be important to assess the effect of MBIs of core symptoms of CMDs, not least because several researchers and theorists have argued that MBIs ought to have positive effects on symptoms of several psychiatric disorders, e.g. social anxiety disorder, hypochondriasis and PTSD (Koszycki et al. 2007; Asmaee Majid et al. 2012; Kearney et al. 2013). Although we consider quality of life to be an important outcome in any psychological treatment (not least as disorder-specific symptoms may remain high while quality of life improves) it was not possible to include such data in the present meta-analysis because only $26 \%$ of the studies reported outcomes related to quality of life.

Further, to be included in the present meta-analysis we required that the individual studies reported outcomes for each patient group separately if the studies included more than one (e.g., studies testing MBIs for depression and anxiety disorders). This is the reason why the previously mentioned study by Sundquist et al. (2015) was not included in our meta-analysis, as it included patients with several different diagnoses and analysed them as one group. If several diagnoses are analysed together, it is not possible to determine for which of the disorders MBIs is effective. In total five studies were excluded for this reason (see online Appendix in the OSM).

To the best of our knowledge, only one previous meta-analysis (Goyal et al. 2014) has evaluated MBIs against criteria for evidencebased treatments. The results from our meta-analysis, at least partly, contradict the conclusions drawn in the study by Goyal et al. where mindfulness meditation programmes were found to have moderate evidence regarding improved anxiety and depression, and low evidence for improving stress/distress (Goyal et al. 2014). There are several possible explanations for these different conclusions. Firstly, Goyal et al. did not focus solely on psychiatric conditions but included studies where the participants had a primary somatic condition/disorder (e.g., HIV, cancer and chronic pain) and where part of the outcomes measured were secondary psychiatric problems (e.g., symptoms of anxiety, depression or stress). Also, it should be noted that the present analysis concerns depression in the acute phase, and not the prevention of relapse, where previous metaanalyses (e.g., Galante et al. 2013; Goyal et al. 2014 and Piet \& Hougaard, 2011) have shown MBCT to be effective. Furthermore, apart from research design (where RCTs are ranked as the highest quality), the evidence criteria applied by Goyal et al. do not take methodological quality into account when grading the evidence (for a review of these criteria, see Owens et al. 2010). As noted above, the criteria developed by the APA task force specifically target several methodological aspects (as described in the methodological criteria, M.1-M.5) that are of great importance when reviewing psychological treatment studies. Also, as the APA criteria are well accepted and widely used within clinical psychological research, we consider it a reasonable choice as this enables comparisons of the evidence base for MBIs with that of other well-established psychological treatments.

\section{Implications for clinical practice}

Whether MBIs is effective for CMDs should be of interest for decision-makers in the healthcare system, as they are responsible for offering evidence-based care for both mild and heterogeneous 
forms of psychiatric ill-health, but also clinically manifest psychiatric disorders. Lack of knowledge on this topic could potentially lead to the risk of patients with CMDs not getting access to effective treatment, i.e. if MBIs would show to be effective in the treatment of CMDs in the acute phase.

The conclusion from this systematic review and meta-analysis is, however, that the available scientific evidence for MBIs in the treatment of acute phases of CMDs varies from low (for hypochondriasis) to no research support (depression, GAD, social anxiety disorder, PTSD, panic disorder, insomnia and elevated stress). Thus, it is not evidence-based care to offer MBIs as a firsthand treatment for CMDs, as there are other treatments that are considered well-established treatments that should be offered, in line with existing guidelines (National Institute for Clinical Excellence, 2011; National Institutes of Health).

\section{Implications for future research}

In light of the methodological pitfalls that was evident in many of the included RCTs, the following recommendations can be made for future studies: (1) Use reliable and valid inclusion procedures in order to generate results that are generalizable to patients with the disorder in question. (2) In studies including patients with different psychiatric disorders, it is vital that the results are presented separately per diagnosis, in order to enable estimation of treatment effect on the patients' principal disorder. (3) Future studies should use active comparisons, e.g. placebo or well-established treatments, and have samples of sufficient size in order to be able to demonstrate non-inferiority, (4) It is of great importance to delineate the treatment protocol in such a detail that the reader could fully understand how the treatment was delivered and to enable replication, (5) In order to determine whether the treatment was delivered according to protocol, future studies should regularly and systematically measure therapist competence and adherence.

\section{Strengths and limitations}

Important strengths of the present systematic review and metaanalyses are the comprehensive literature search and the thorough assessment of study quality, the risk of bias and of the evidence status. A specific strength was that we both calculated the aggregated effects across all disorders, and also conducted disorderspecific evidence assessment. This means that potential differences in effects of MBIs for different disorders could be detected. Limitations are that we were not able to conduct moderator analyses on all variables, due to the paucity of available data on these variables. It would have been preferred if all disorders could have been compared across all available comparison groups (i.e., WLC, TAU, placebo and active treatments including CBT and pharmacological treatment), but this was not possible due to the low number of studies included. Furthermore, the choice to exclude studies in other languages than the English infers the risk that we missed studies possibly eligible for inclusion.

\section{Conclusion}

We conclude that the evidence basis for MBIs in the treatment of CMDs in the acute phase is weak and that it should not be used as a first-hand treatment option. This systematic review and meta-analysis have important implications for treatment guidelines concerning MBIs.

\section{Supplementary material}

The supplementary material for this article can be found at https://doi.org/10.1017/S0033291718000259.

Acknowledgement. This work was supported by Stockholm County Council.

\section{Declaration of interest}

None.

\section{References}

American Psychological Association, S. o. c. p. (2016) Research-Supported Psychological Treatments. American Psychological Association, Society of Clinical Psychology. Available at http://www.div12.org/psychological-treatments/.

Asmaee Majid S, Seghatoleslam T, Homan H, Akhvast A and Habil H (2012) Effect of mindfulness based stress management on reduction of generalized anxiety disorder. Iranian Journal of Public Health 41, 24-28.

Baer RA (2003) Mindfulness training as a clinical intervention: a conceptual and empirical review. Clinical Psychology: Science and Practice 10, 125-143.

Balshem H, Helfand M, Schünemann HJ, Oxman AD, Kunz R, Brozek J et al. (2011) GRADE guidelines: 3. Rating the quality of evidence. Journal of Clinical Epidemiology 64, 401-406.

Barnhofer T, Crane C, Hargus E, Amarasinghe M, Winder R and Williams JM (2009) Mindfulness-based cognitive therapy as a treatment for chronic depression: a preliminary study. Behaviour Research and Therapy 47, 366-373.

Biegel GM, Brown KW, Shapiro SL and Schubert CM (2009) Mindfulnessbased stress reduction for the treatment of adolescent psychiatric outpatients: a randomized clinical trial. Journal of Consulting and Clinical Psychology 77, 855-866.

Black DS, O'Reilly GA, Olmstead R, Breen EC and Irwin MR (2015) Mindfulness meditation and improvement in sleep quality and daytime impairment among older adults with sleep disturbances: a randomized clinical trial. JAMA Internal Medicine 175, 494-501.

Bower JE, Crosswell AD, Stanton AL, Crespi CM, Winston D, Arevalo J et al. (2015) Mindfulness meditation for younger breast cancer survivors: a randomized controlled trial. Cancer 121, 1231-1240.

Bränström R, Kvillemo P and Moskowitz JT (2012) A randomized study of the effects of mindfulness training on psychological well-being and symptoms of stress in patients treated for cancer at 6-month follow-up. International Journal of Behavioral Medicine 19, 535-542.

Brown KW, Ryan RM and Creswell JD (2007) Mindfulness: theoretical foundations and evidence for its salutary effects. Psychological Inquiry 18, 211237.

Chambless DL, Baker MJ, Baucom DH, Beutler LE, Calhoun KS, Crits-Christoph P et al. (1998) Update on empirically validated therapies, II. The Clinical Psychologist 51, 3-16.

Chiesa A, Castagner V, Andrisano C, Serretti A, Mandelli L, Porcelli S et al. (2015) Mindfulness-based cognitive therapy vs. psycho-education for patients with major depression who did not achieve remission following antidepressant treatment. Psychiatry Research 226, 474-483.

Chiesa A and Serretti A (2011) Mindfulness based cognitive therapy for psychiatric disorders: a systematic review and meta-analysis. Psychiatry Research 187, 441-453.

Degenhardt L, Whiteford HA, Ferrari AJ, Baxter AJ, Charlson FJ, Hall WD et al. (2013) Global burden of disease attributable to illicit drug use and dependence: findings from the global burden of disease study 2010. The Lancet 382, 1564-1574.

Delgado LC, Guerra P, Perakakis P, Vera MN, Reyes del Paso G and Vila J (2010) Treating chronic worry: psychological and physiological effects of a training programme based on mindfulness. Behaviour Research and Therapy 48, 873-882.

Demarzo MM, Cebolla A and Garcia-Campayo J (2015a) The implementation of mindfulness in healthcare systems: a theoretical analysis. General Hospital Psychiatry 37, 166-171. 
Demarzo MM, Montero-Marin J, Cuijpers P, Zabaleta-del-Olmo E, Mahtani KR, Vellinga A et al. (2015b) The efficacy of mindfulness-based interventions in primary care: a meta-analytic review. Annals of Family Medicine 13, 573-582.

Dimidjian S and Segal ZV (2015) Prospects for a clinical science of mindfulness-based intervention. American Psychologist 70, 593-620.

Duval S and Tweedie R (2000) Trim and fill: a simple funnel-plot-based method of testing and adjusting for publication bias in meta-analysis. Biometrics 56, 455-463.

Egger M, Davey Smith G, Schneider M and Minder C (1997) Bias in meta-analysis detected by a simple, graphical test. British Medical Journal $315,629-634$.

Eisendrath SJ, Gillung E, Delucchi KL, Segal ZV, Nelson JC, McInnes LA et al. (2016) A randomized controlled trial of mindfulness-based cognitive therapy for treatment-resistant depression. Psychotherapy and Psychosomatics 85, 99-110.

Galante J, Iribarren SJ and Pearce PF (2013) Effects of mindfulness-based cognitive therapy on mental disorders: a systematic review and meta-analysis of randomised controlled trials. Journal of Research in Nursing 18, 133-155.

Goyal M, Singh S, Sibinga EM, Gould NF, Rowland-Seymour A, Sharma R et al. (2014) Meditation programs for psychological stress and well-being: a systematic review and meta-analysis. JAMA Internal Medicine 174, 357-368.

Gross CR, Kreitzer MJ, Thomas W, Reilly-Spong M, CramerBornemann M, Nyman JA et al. (2010) Mindfulness-based stress reduction for solid organ transplant recipients: a randomized controlled trial. Alternative Therapies in Health and Medicine 16, 30-38.

Grossman P, Niemann L, Schmidt S and Walach H (2004) Mindfulnessbased stress reduction and health benefits. A meta-analysis. Journal of Psychosomatic Research 57, 35-43.

Guardino CM, Dunkel Schetter C, Bower JE, Lu MC and Smalley SL (2014) Randomised controlled pilot trial of mindfulness training for stress reduction during pregnancy. Psychology \& Health 29, 334-349.

Hamidian S, Omidi A, Mousavinasab SM and Naziri G (2013) Comparison of the effect of mindfulness-based cognitive therapy accompanied by pharmacotherapy with pharmacotherapy alone in treating dysthymic patients. Iranian Red Crescent Medical Journal 15, 239-244.

Hedges LV (1982) Estimation of effect size from a series of independent experiments. Psychological Bulletin 92, 490-499.

Higgins JP, Altman DG, Gotzsche PC, Juni P, Moher D, Oxman AD et al. Cochrane Bias Methods, G., Cochrane Statistical Methods, G. (2011) The Cochrane Collaboration's tool for assessing risk of bias in randomised trials. British Medical Journal 343, d5928.

Hofmann SG, Sawyer AT, Witt AA and Oh D (2010) The effect of mindfulness-based therapy on anxiety and depression: a meta-analytic review. Journal of Consulting and Clinical Psychology 78, 169-183.

Hoge EA, Bui E, Marques L, Metcalf CA, Morris LK, Robinaugh DJ et al. (2013) Randomized controlled trial of mindfulness meditation for generalized anxiety disorder: effects on anxiety and stress reactivity. The Journal of Clinical Psychiatry 74, 786-792.

Jadad AR, Moore A, Carroll D, Jenkinson C, Reynolds JM, Gavaghan DJ et al. (1996) Assessing the quality of reports of randomized clinical trials: is blinding necessary? Controlled Clinical Trials 17, 1-12.

Jazaieri H, Goldin PR, Werner K, Ziv M and Gross JJ (2012) A randomized trial of MBSR versus aerobic exercise for social anxiety disorder. Journal of Clinical Psychology 68, 715-731.

Kabat-Zinn J (1996) Full Catastrophe Living : How to Cope with Stress, Pain and Illness Using Mindfulness Meditation. London: Piatkus.

Kearney DJ, McDermott K, Malte C, Martinez M and Simpson TL (2013) Effects of participation in a mindfulness program for veterans with posttraumatic stress disorder: a randomized controlled pilot study. Journal of Clinical Psychology 69, 14-27.

Khoury B, Lecomte T, Fortin G, Masse M, Therien P, Bouchard V et al. (2013) Mindfulness-based therapy: a comprehensive meta-analysis. Clinical Psychology Review 33, 763-771.

Koszycki D, Benger M, Shlik J and Bradwejn J (2007) Randomized trial of a meditation-based stress reduction program and cognitive behavior therapy in generalized social anxiety disorder. Behaviour Research and Therapy 45, 2518-2526.
Lengacher CA, Johnson-Mallard V, Post-White J, Moscoso MS, Jacobsen PB, Klein TW et al. (2009) Randomized controlled trial of mindfulness-based stress reduction (MBSR) for survivors of breast cancer. Psycho-oncology 18, 1261-1272.

Lenz AS, Hall J and Bailey Smith L (2016) Meta-analysis of group mindfulness-based cognitive therapy for decreasing symptoms of acute depression. Journal for Specialists in Group Work 41, 44-70.

Lipsey MW and Wilson D (2001) Practical Meta-Analysis. London: SAGE.

Ludwig DS and Kabat-Zinn J (2008) Mindfulness in medicine. JAMA $\mathbf{3 0 0}$, $1350-1352$.

McManus F, Surawy C, Muse K, Vazquez-Montes M and Williams JM (2012) A randomized clinical trial of mindfulness-based cognitive therapy versus unrestricted services for health anxiety (hypochondriasis). Journal of Consulting and Clinical Psychology 80, 817-828.

Michalak J, Schultze M, Heidenreich T and Schramm E (2015) A randomized controlled trial on the efficacy of mindfulness-based cognitive therapy and a group version of cognitive behavioral analysis system of psychotherapy for chronically depressed patients. Journal of Consulting and Clinical Psychology 83, 951-963.

Moher D, Liberati A, Tetzlaff J and Altman DG (2009) Preferred reporting items for systematic reviews and meta-analyses: the PRISMA statement PLOS Medicine 151, 264-269, W64.

National Institute for Clinical Excellence (2011) Generalised Anxiety Disorder and Panic Disorder (with or without Agoraphobia) in Adults: Management in Primary, Secondary and Community Care. London: National Collaborating Centre for Mental Health.

National Institutes of Health (2017) U.S. Department of Health \& Human Services. Bethesda, MD: National Institutes of Health, USA.gov.

Ong JC, Manber R, Segal Z, Xia Y, Shapiro S and Wyatt JK (2014) A randomized controlled trial of mindfulness meditation for chronic insomnia. Sleep 37, 1553-1563.

Öst L-G (2008) Efficacy of the third wave of behavioral therapies: a systematic review and meta-analysis. Behaviour Research and Therapy 46, 296-321.

Öst L-G (2014) The efficacy of acceptance and commitment therapy: an updated systematic review and meta-analysis. Behaviour Research and Therapy 61, 105-121.

Öst L-G, Havnen A, Hansen B and Kvale G (2015) Cognitive behavioral treatments of obsessive-compulsive disorder. A systematic review and meta-analysis of studies published 1993-2014. Clinical Psychology Review 40, 156-169.

Öst L-G, Riise EN, Wergeland GJ, Hansen B and Kvale G (2016) Cognitive behavioral and pharmacological treatments of OCD in children: a systematic review and meta-analysis. Journal of Anxiety Disorders 43, 58-69.

Owens DK, Lohr KN, Atkins D, Treadwell JR, Reston JT, Bass EB et al. (2010) AHRQ series paper 5: grading the strength of a body of evidence when comparing medical interventions - agency for healthcare research and quality and the effective health-care program. Journal of Clinical Epidemiology 63, 513-523.

Piet J and Hougaard E (2011) The effect of mindfulness-based cognitive therapy for prevention of relapse in recurrent major depressive disorder: a systematic review and meta-analysis. Clinical Psychology Review 31, 1032-1040.

Piet J, Hougaard E, Hecksher MS and Rosenberg NK (2010) A randomized pilot study of mindfulness-based cognitive therapy and group cognitivebehavioral therapy for young adults with social phobia. Scandinavian Journal of Psychology 51, 403-410.

Plank K (2010) Mindful medicine: the growing trend of mindfulness-based therapies in the Swedish health care system. Finnish Journal of Ethnicity and Migration 5, 47-55.

Polusny MA, Erbes CR, Thuras P, Moran A, Lamberty GJ, Collins RC et al. (2015) Mindfulness-based stress reduction for posttraumatic stress disorder among veterans: a randomized clinical trial. JAMA 314, 456-465.

Possemato K, Bergen-Cico D, Treatman S, Allen C, Wade M and Pigeon W (2015) A randomized clinical trial of primary care brief mindfulness training for veterans With PTSD. Journal of Clinical Psychology 72, 179-193.

Segal ZV, Bieling P, Young T, MacQueen G, Cooke R, Martin L et al. (2010) Antidepressant monotherapy $v s$ sequential pharmacotherapy and mindfulness-based cognitive therapy, or placebo, for relapse prophylaxis in recurrent depression. Archives of General Psychiatry 67, 1256-1264. 
Shapiro DA and Shapiro D (1982) Meta-analysis of comparative therapy outcome studies: A replication and refinement. Psychological Bulletin 92, 581-604.

Southam-Gerow MA and Prinstein MJ (2014) Evidence base updates: the evolution of the evaluation of psychological treatments for children and adolescents. Journal of Clinical Child \& Adolescent Psychology 43, 1-6.

Strauss C, Cavanagh K, Oliver A and Pettman D (2014) Mindfulness-based interventions for people diagnosed with a current episode of an anxiety or depressive disorder: a meta-analysis of randomised controlled trials. PLoS ONE 9, e96110.

Sundquist J, Lilja A, Palmer K, Memon AA, Wang X, Johansson LM et al. (2015) Mindfulness group therapy in primary care patients with depression, anxiety and stress and adjustment disorders: randomised controlled trial. The British Journal of Psychiatry 206, 128-135.

Teasdale JD, Segal ZV, Williams JM, Ridgeway VA, Soulsby JM and Lau MA (2000) Prevention of relapse/recurrence in major depression by mindfulness-based cognitive therapy. Journal of Consulting and Clinical Psychology 68, 615-623.

van Aalderen JR, Donders AR, Giommi F, Spinhoven P, Barendregt HP and Speckens AE (2012) The efficacy of mindfulness-based cognitive therapy in recurrent depressed patients with and without a current depressive episode: a randomized controlled trial. Psychological Medicine 42, 989-1001.
Vollestad J, Nielsen MB and Nielsen GH (2012) Mindfulness- and acceptance-based interventions for anxiety disorders: a systematic review and meta-analysis. British Journal of Clinical Psychology 51, 239-260.

Wahbeh H, Goodrich E, Goy E and Oken BS (2016) Mechanistic pathways of mindfulness meditation in combat veterans With posttraumatic stress disorder. Journal of Clinical Psychology 72, 365-383.

Wampold BE, Mondin GW, Moody M, Stich F, Benson K and Ahn H-n (1997) A meta-analysis of outcome studies comparing bona fide psychotherapies: Empiricially, “all must have prizes." Psychological Bulletin 122, 203-215.

Weisz JR, Weiss B, Han SS, Granger DA and Morton T (1995) Effects of psychotherapy with children and adolescents revisited: A meta-analysis of treatment outcome studies. Psychological Bulletin 117, 450-468.

Williams JM, Crane C, Barnhofer T, Brennan K, Duggan DS, Fennell MJ et al. (2014) Mindfulness-based cognitive therapy for preventing relapse in recurrent depression: a randomized dismantling trial. Journal of Consulting and Clinical Psychology 82, 275-286.

Zhang JX, Liu XH, Xie XH, Zhao D, Shan MS, Zhang XL et al. (2015) Mindfulness-based stress reduction for chronic insomnia in adults older than 75 years: a randomized, controlled, single-blind clinical trial. Explore (NY) 11, 180-185. 